\title{
Pin-Collinear Body-and-Pin Frameworks and the Molecular Conjecture
}

\author{
Bill Jackson · Tibor Jordán
}

Received: 31 October 2006 / Revised: 5 July 2008 / Accepted: 5 July 2008 /

Published online: 28 August 2008

(C) Springer Science+Business Media, LLC 2008

\begin{abstract}
T.-S. Tay and W. Whiteley independently characterized the multigraphs which can be realized as an infinitesimally rigid $d$-dimensional body-and-hinge framework. In 1984 they jointly conjectured that each graph in this family can be realized as an infinitesimally rigid framework with the additional property that the hinges incident to each body lie in a common hyperplane. This conjecture has become known as the Molecular Conjecture because of its implication for the rigidity of molecules in 3-dimensional space. Whiteley gave a partial solution for the 2-dimensional form of the conjecture in 1989 by showing that it holds for multigraphs $G=(V, E)$ in the family which have the minimum number of edges, i.e. satisfy $2|E|=3|V|-3$. In this paper, we give a complete solution for the 2-dimensional version of the Molecular Conjecture. Our proof relies on a new formula for the maximum rank of a pincollinear body-and-pin realization of a multigraph as a 2-dimensional bar-and-joint framework.
\end{abstract}

Keywords Rigidity of frameworks · Body-and-hinge frameworks $\cdot$ Bar-and-joint frameworks · Molecular Conjecture

This work was supported by an International Joint Project grant of the Royal Society. The research of T. Jordán was also supported by the MTA-ELTE Egerváry Research Group on Combinatorial Optimization and the Hungarian Scientific Research Fund grant no. T49671.

B. Jackson $(\bowtie)$

School of Mathematical Sciences, Queen Mary, University of London, Mile End Road, London E1 4NS, England, UK

e-mail: b.jackson@qmul.ac.uk

T. Jordán

Department of Operations Research, Eötvös University, Pázmány Péter sétány 1/C, 1117 Budapest, Hungary

e-mail: jordan@cs.elte.hu 


\section{Introduction}

Informally, a body-and-hinge framework in $\mathbb{R}^{d}$ consists of large rigid bodies articulated along affine subspaces of dimension $d-2$ which act as hinges, i.e. bodies joined by pin-joints in 2-space, line-hinges in 3-space, plane-hinges in 4-space, etc. The bodies are free to move continuously in $\mathbb{R}^{d}$ subject to the constraint that the relative motion of any two bodies joined by a hinge is a rotation about the hinge. The framework is rigid if every such motion preserves the distances between all pairs of points belonging to different rigid bodies, i.e. the motion extends to an isometry of $\mathbb{R}^{d}$. The incidences can be described by a multigraph $G$ in which vertices correspond to bodies and edges correspond to hinges. The framework is said to be a realization of $G$ in $\mathbb{R}^{d}$.

The rigidity of a $d$-dimensional body-and-hinge framework can be investigated using an associated rigidity matrix. The framework is infinitesimally rigid if this matrix has rank $\left(\begin{array}{c}d+1 \\ 2\end{array}\right)(|V(G)|-1)$, and this is known to be a sufficient condition for the rigidity of the framework. We refer the reader to $[5,14,16]$ for formal definitions and a more detailed account of body-and-hinge frameworks in $\mathbb{R}^{d}$. We will only be concerned with the case $d=2$ and use a different but equivalent way to model the body-and-hinge frameworks we are dealing with.

Multigraphs ${ }^{1}$ which can be realized as infinitesimally rigid body-and-hinge frameworks are characterized by the following theorem proved independently by Tay and Whiteley. Given a multigraph $G$ and a positive integer $k$, we use $k G$ to denote the multigraph obtained by replacing each edge of $G$ by $k$ parallel edges.

Theorem 1.1 [11, 14] A multigraph $G$ can be realized as an infinitesimally rigid body-and-hinge framework in $\mathbb{R}^{d}$ if and only if $\left(\left(\begin{array}{c}d+1 \\ 2\end{array}\right)-1\right) G$ has $\left(\begin{array}{c}d+1 \\ 2\end{array}\right)$ edge-disjoint spanning trees.

Tay and Whiteley jointly made the following conjecture which would strengthen their theorem.

Conjecture 1.2 [12] Let $G$ be a multigraph. Then $G$ can be realized as an infinitesimally rigid body-and-hinge framework in $\mathbb{R}^{d}$ if and only if $G$ can be realized as an infinitesimally rigid body-and-hinge framework in which the hinges incident to each body are contained in a common hyperplane.

The purpose of this paper is to give a complete solution of this conjecture when $d=2$. We will assume henceforth that $d=2$, unless stated otherwise, and refer to hinges as pins and to body-and-hinge frameworks as body-and-pin frameworks. We say that a body-and-pin framework is pin-collinear if the pins incident to each body lie on a common line. We shall prove:

\footnotetext{
${ }^{1}$ All graphs considered are finite and without loops. We will reserve the term graph for graphs without multiple edges and refer to graphs which may contain multiple edges as multigraphs.
} 

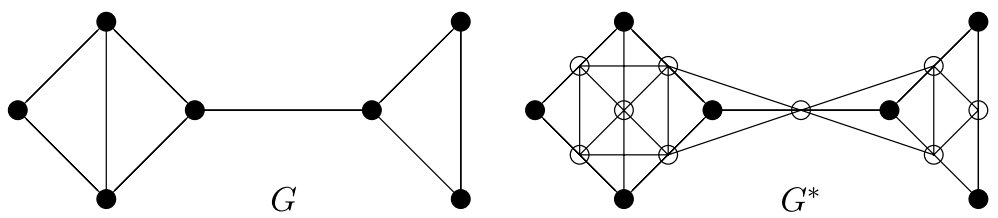

Fig. 1 A graph $G$ and its body-pin graph $G^{*}$. The vertices of $G$ represent bodies, and the edges of $G$ represent pins connecting bodies. In $G^{*}$ each vertex of $G$ is replaced by a complete graph (body), and each edge of $G$ becomes a shared vertex (pin) between two complete graphs

Theorem 1.3 Let $G$ be a multigraph. Then $G$ has an infinitesimally rigid pincollinear body-and-pin realization if and only if $2 G$ contains three edge-disjoint spanning trees.

Theorem 1.3 has been verified by Whiteley [15], by using a different approach, in the special case where $2 G$ is the union of three edge-disjoint spanning trees. In this special case an infinitesimally rigid realization of $G$ is necessarily "independent" in the sense that there is no redundancy in the constraints on the infinitesimal motions of the framework. It is important to note that the general case is far from being an easy extension of the "independent" result. The existence of three edge-disjoint spanning trees in $2 G$ does not imply that $G$ has a spanning subgraph $H$ for which $2 H$ is the union of three edge-disjoint spanning trees. To see this, consider for example a graph $G$ with an even number of vertices. The existence of redundancy in the constraints make the general problem substantially more difficult. More remarks on this aspect of the problem will be given at the end of Sect. 5 .

Our proof of Theorem 1.3 uses an equivalent model of body-and-pin structures as 'bar-and-joint' frameworks. The equivalence is based on the fact, which is intuitively clear and not difficult to verify (see [2]), that the "degree of freedom" of the framework does not change if we replace the rigid bodies by rigid bar-and-joint frameworks in such a way that each pin becomes a pin-joint. The underlying graph of this framework can be defined as follows.

Let $G=(V, P)$ be a multigraph. For $v \in V$, let $E_{G}(v)$ be the set of all edges of $G$ incident to $v$. The body-and-pin graph of $G$ is the graph $G^{*}$ with $V\left(G^{*}\right)=V \cup P$ and

$$
E\left(G^{*}\right)=\left\{v p: v \in V \text { and } p \in E_{G}(v)\right\} \cup\left\{p_{1} p_{2}: v \in V \text { and } p_{1}, p_{2} \in E_{G}(v)\right\}
$$

Thus $G^{*}$ is obtained from $G$ by first subdividing each edge of $G$ and then adding a complete graph on the new neighbour set of each vertex $v \in V$, see Fig. 1.

A (2-dimensional) bar-and-joint framework $(H, q)$ is a graph $H=(V, E)$ together with a map $q: V \rightarrow \mathbb{R}^{2}$. We say that $(H, q)$ is a realization of $H$. We consider each vertex $v \in V$ to be represented by a universal joint at $q(v)$ and each edge $e=u v \in E$ to be represented by a rigid bar attached to the joints $q(u)$ and $q(v)$. Let $G=(V, P)$ be a multigraph and $G^{*}$ be the body-and-pin graph of $G$. Given a map $q: V\left(G^{*}\right) \rightarrow \mathbb{R}^{2}$, we say that the bar-and-joint framework $\left(G^{*}, q\right)$ is a body-and-pin realization of $G$ if $q$ acts injectively on $\{v\} \cup E_{G}(v)$ for all $v \in V$ and the subframe- 
work of $\left(G^{*}, q\right)$ induced by $\{v\} \cup E_{G}(v)$ is infinitesimally rigid. ${ }^{2}$ The realization $\left(G^{*}, q\right)$ is said to be pin-collinear if the points $q(p), p \in E_{G}(v)$, are collinear for each $v \in V$. We will deduce Theorem 1.3 from the following equivalent result.

Theorem 1.4 Let $G$ be a multigraph. Then $G$ has an infinitesimally rigid pincollinear body-and-pin realization as a bar-and-joint framework if and only if $2 G$ has three edge-disjoint spanning trees.

In fact, we shall determine the maximum rank (or equivalently, the minimum degree of freedom) of the pin-collinear body-and-pin realizations of a multigraph as a bar-and-joint framework. To state and prove this more general result we need some concepts and results on forest covers of multigraphs, which will be described in Sect. 3. The proof of our main result will follow in Sect. 5. We conclude the paper by describing a conjectured characterization of when a general incidence structure can be realized as an infinitesimally rigid pin-collinear body-and-pin framework in Sect. 6.

\section{The Molecular Conjecture}

The main motivation of our work is to develop new methods which can take us closer to the solution of the 3-dimensional case of Conjecture 1.2. This special case is known as the Molecular Conjecture because it has important implications for the rigidity of molecules. Under projective duality in $\mathbb{R}^{3}$, lines are mapped to lines. Hence one may define the dual of a 3-dimensional body-and-hinge framework by replacing the line associated with each hinge by its projective dual. Crapo and Whiteley [1] show that the infinitesimal rigidity of a body-and-hinge framework is invariant under this duality. Since projective duality also interchanges planes and points, the statement that a graph $G$ can be realized as an infinitesimally rigid body-and-hinge framework in $\mathbb{R}^{3}$ with all hinges incident to each body coplanar is equivalent to the statement that $G$ can be realized as an infinitesimally rigid body-and-hinge framework in $\mathbb{R}^{3}$ with all hinges incident to each body concurrent at a point. The application to molecules constructs a body-and-hinge framework which represents each atom as a body. The relative motion of two atoms which are linked by a bond is constrained to be a rotation about the line through the centre of the atoms. This gives rise to a body-and-hinge framework in which all hinges incident to a body are concurrent $[17,18]$. The truth of the Molecular Conjecture would imply that the results on generic body-and-hinge frameworks (e.g. the characterization of rigidity, algorithms for computing the degree of freedom, etc.) extend to such molecular frameworks. For partial results on the Molecular Conjecture and equivalent versions in terms of bar-and-joint frameworks, see $[3-5,18]$.

We remark that Theorem 1.3 also has an implication for the Molecular Conjecture. Suppose that $2 G$ has three edge-disjoint spanning trees and let us consider an infinitesimally rigid pin-collinear body-and-pin realization of $G$ in the plane. One may use it to construct an infinitesimally rigid 3-dimensional body-and-hinge realization of

\footnotetext{
${ }^{2} \mathrm{~A}$ formal definition of the infinitesimal rigidity of bar-and-joint frameworks will be given in Sect. 2 .
} 
$G$ in which the pins become vertical hinge lines which are concurrent at a projective point at infinity. In addition all hinges incident to a given body will be contained in the same vertical plane (containing the corresponding planar pin-line). The projective dual will then be an infinitesimally rigid 3-dimensional body-and-hinge realization of $G$ with all hinges incident to each body concurrent, as well as all hinges overall coplanar. (A partial extension of this result to all dimensions is given in [5], where we show that if $(d-1) G$ has $d$ edge-disjoint spanning trees, then $G$ can be realized as an infinitesimally rigid $d$-dimensional body-and-hinge framework with all its hinges contained in the same hyperplane.)

\section{Bar-and-Joint Frameworks}

For completeness, we state the basic lemmas we need on 2-dimensional frameworks. Since these lemmas are either well known or easy to prove, we omit the proofs. A reader familiar with rigidity of frameworks may skip this section.

Let $G=(V, E)$ be a graph, and let $(G, q)$ be a bar-and-joint framework in $\mathbb{R}^{2}$. The rigidity matrix of the framework is the matrix $R(G, q)$ of size $|E| \times 2|V|$, where, for each edge $v_{i} v_{j} \in E$, in the row corresponding to $v_{i} v_{j}$, the entries in the two columns corresponding to vertices $v_{i}$ and $v_{j}$ are given by the coordinates of $q\left(v_{i}\right)-q\left(v_{j}\right)$ and $q\left(v_{j}\right)-q\left(v_{i}\right)$, respectively, and the remaining entries are zeros. See [16] for more details. We denote the rank of $R(G, q)$ by $r(G, q)$ and refer to it as the rank of the framework. The degree of freedom of the framework is defined to be $2|V|-r(G, q)$, i.e. the dimension of the null space of $R(G, q)$. The following lemma gives an upper bound on $r(G, q)$.

Lemma 2.1 [16, Lemma 11.1.3] Let $(G, q)$ be a bar-and-joint framework with $n \geq 2$ vertices. Then $r(G, q) \leq 2 n-3$.

A framework $(G, q)$ is said to be infinitesimally rigid if $r(G, q)=2 n-3$. The following lemma follows from the fact that the entries in $R(G, q)$ are polynomial (and hence continuous) functions of the components of $q(v), v \in V$.

Lemma 2.2 Let $(G, q)$ be a bar-and-joint framework. Then there exists $\epsilon>0$ such that for all $q^{\prime}: V \rightarrow \mathbb{R}^{2}$ with $\left\|q^{\prime}(v)-q(v)\right\| \leq \epsilon$ for all $v \in V$, we have $r\left(G, q^{\prime}\right) \geq$ $r(G, q)$.

Let $G=(V, E)$ be a multigraph. For $X \subseteq V$, the degree of $X, d_{G}(X)$, is the number of edges of $G$ from $X$ to $V-X$. If $X=\{v\}$ for some $v \in V$, then we simply write $d_{G}(v)$ for the degree of $v$. The set of neighbours of $X$ (i.e. the set of those vertices $v \in V-X$ for which there exists an edge $u v \in E$ with $u \in X$ ) is denoted by $N_{G}(X)$.

Lemma 2.3 [16, Lemma 2.1.3] Let $G_{1}=\left(V_{1}, E_{1}\right)$ be a graph and $v_{1}$, $v_{2}$ be distinct vertices of $G_{1}$. Let $G$ be obtained from $G_{1}$ by adding a new vertex $v$ and edges $v v_{1}, v v_{2}$. Let $\left(G_{1}, q_{1}\right)$ be a realization of $G_{1}$ such that $q_{1}\left(v_{1}\right) \neq q_{1}\left(v_{2}\right)$. Choose a 
point $Q$ such that $q_{1}\left(v_{1}\right), q_{1}\left(v_{2}\right), Q$ are not collinear, and let $(G, q)$ be the realization of $G$ obtained from $\left(G_{1}, q_{1}\right)$ by putting $q(v)=Q$. Then $r(G, q)=r\left(G_{1}, q_{1}\right)+2$.

Lemma 2.4 [16, Lemma 2.2.2] Let $G_{1}=\left(V_{1}, E_{1}\right)$ be a graph, $v_{1}, v_{2}$, $v_{3}$ be distinct vertices of $G_{1}$ and $v_{1} v_{2}$ be an edge of $G_{1}$. Let $G$ be obtained from $G_{1}-v_{1} v_{2}$ by adding a new vertex $v$ and edges $v v_{1}, v v_{2}, v v_{3}$. Let $\left(G_{1}, q_{1}\right)$ be a realization of $G_{1}$ such that $q_{1}\left(v_{1}\right), q_{1}\left(v_{2}\right), q_{1}\left(v_{3}\right)$ are not collinear. Let $Q \in \mathbb{R}^{2}$ be a point on the line through $q_{1}\left(v_{1}\right), q_{1}\left(v_{2}\right)$ distinct from $q_{1}\left(v_{1}\right), q_{1}\left(v_{2}\right)$. Let $(G, q)$ be the realization of $G$ obtained from $\left(G_{1}, q_{1}\right)$ by putting $q(v)=Q$. Then $r(G, q)=r\left(G_{1}, q_{1}\right)+2$.

The following result is given without proof in [16, Fig. 2.9].

Lemma 2.5 Let $G=(V, E)$ be a graph, $v \in V$ and $E_{G}(v)=\left\{v v_{1}, v v_{2}, \ldots, v v_{k}\right\}$ for some $k \geq 2$. Choose $j$ such that $2 \leq j \leq k$, and let $G^{\prime}$ be the graph obtained from $G-v$ by adding two new vertices $v^{\prime}, v^{\prime \prime}$ and edges $v^{\prime} v_{1}, v^{\prime} v_{2}, \ldots, v^{\prime} v_{j}, v^{\prime \prime} v_{1}, v^{\prime \prime} v_{2}$, $v^{\prime \prime} v_{j+1}, \ldots, v^{\prime \prime} v_{k}$. Suppose that $q: V \rightarrow \mathbb{R}^{2}$. Define $q^{\prime}: V\left(G^{\prime}\right) \rightarrow \mathbb{R}^{2}$ by $q^{\prime}(u)=$ $q(u)$ for all $u \in V-v$ and $q\left(v^{\prime}\right)=q\left(v^{\prime \prime}\right)=q(v)$. Suppose that $q(v)-q\left(v_{1}\right)$ and $q(v)-q\left(v_{2}\right)$ are linearly independent. Then $r\left(G^{\prime}, q^{\prime}\right) \geq r(G, q)+2$.

We refer to the operations in Lemmas 2.3, 2.4 and 2.5 as 0-extensions, 1-extensions and vertex-splits, respectively. See Figs. 2 and 3.

Lemma 2.6 Let $G=(V, E), G_{1}=\left(V_{1}, E_{1}\right), G_{2}=\left(V_{2}, E_{2}\right)$ be connected graphs such that $G=G_{1} \cup G_{2}$, and $G_{1} \cap G_{2}=K$ is a complete graph on $t$ vertices for $t \in\{0,1,2\}$. Let $(G, q)$ be a realization of $G$, and let $q_{i}$ be the restriction of $q$ to $V_{i}$, $i \in\{1,2\}$. Suppose that the affine hull of $q_{i}\left(V_{i}\right)$ is 2-dimensional for each $i \in\{1,2\}$ and that $q(u) \neq q(v)$ if $u, v$ are distinct vertices of $K$. Then $r(G, q)=r\left(G_{1}, q_{1}\right)+$ $r\left(G_{2}, q_{2}\right)-|E(K)|$.

Fig. 2 The graphs $G_{2}$ and $G_{3}$ are the underlying graphs of the frameworks obtained from a realization of $G_{1}$ by a 0 -extension and a 1-extension, respectively

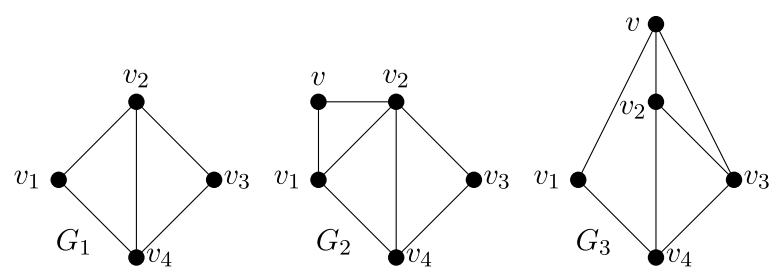

Fig. 3 The vertex-split operation
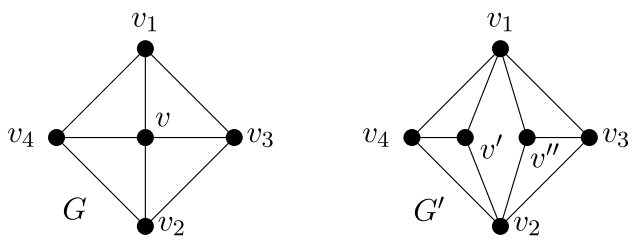


\section{Bricks and Superbricks}

A high-level description of our proof of Theorem 1.4 is as follows. We prove a more general result on the maximum rank of a pin-collinear body-and-pin realization $\left(G^{*}, q\right)$ of a graph $G$. This allows us to use local operations in our inductive proof, which verifies the rank formula by induction on the size of $G$. The proof has a strong graph theoretical flavour but also uses geometric arguments. As we shall see, the maximum rank of $\left(G^{*}, q\right)$ depends on the 'deficiency' of $G$, which measures how far $2 G$ is from having three edge-disjoint spanning trees. To work with this deficiency function we need a number of results on forest covers of graphs from [6]. These lemmas will be given in this section. In our inductive argument we shall apply local or global operations to $G$ (for example edge or vertex deletion, restriction to a connected component) to obtain smaller graphs. We give bounds on the deficiency of the smaller graphs by using our lemmas on forest covers. By induction, this will give us pin-collinear realizations of the smaller graphs of sufficiently high rank. To obtain the desired maximum rank realization of $G^{*}$ we shall apply the geometric lemmas from the previous section.

We now summarize the structural results on forest covers of multigraphs that we shall use. Let $H=(V, E)$ be a multigraph. For a family $\mathcal{F}$ of pairwise disjoint subsets of $V$, let $E_{H}(\mathcal{F})$ denote the set, and $e_{H}(\mathcal{F})$ the number, of edges of $H$ connecting distinct members of $\mathcal{F}$.

The following theorem of Nash-Williams and Tutte is well known, see also [10, Chap. 51].

Theorem 3.1 $[8,9,13]$ Let $H=(V, E)$ be a multigraph, and let $k$ be a positive integer. Then

(a) the maximum size of the union of $k$ forests in $H$ is equal to the minimum value of

$$
e_{H}(\mathcal{P})+k(|V|-|\mathcal{P}|)
$$

taken over all partitions $\mathcal{P}$ of $V$.

(b) $H$ contains $k$ edge-disjoint spanning trees if and only if

$$
e_{H}(\mathcal{P}) \geq k(|\mathcal{P}|-1)
$$

for all partitions $\mathcal{P}$ of $V$.

In this paper we shall be concerned with the case where $H=2 G$ for some multigraph $G=(V, E)$ and $k=3$. For a partition $\mathcal{Q}$ of $V$, let

$$
\operatorname{def}_{G}(\mathcal{Q})=3(|\mathcal{Q}|-1)-2 e_{G}(\mathcal{Q})
$$

denote the deficiency of $\mathcal{Q}$ in $G$, and let

$$
\operatorname{def}(G)=\max \left\{\operatorname{def}_{G}(\mathcal{Q}): \mathcal{Q} \text { is a partition of } V\right\} .
$$

Note that $\operatorname{def}(G) \geq 0 \operatorname{since}^{\operatorname{def}_{G}}(\{V\})=0$. We say that a partition $\mathcal{Q}$ of $V$ is a tight partition of $G$ if $\operatorname{def}_{G}(\mathcal{Q})=\operatorname{def}(G)$. 
We next show that $\operatorname{def}(G)$ can be used to obtain an upper bound on the rank of any bar-and-joint realization $r\left(G^{*}, q\right)$ of the body-and-pin graph of $G$.

Lemma 3.2 Let $G=(V, P)$ be a multigraph with no isolated vertices. Then $r\left(G^{*}, q\right) \leq 2(|V|+|P|)-3-\operatorname{def}(G)$ for all realizations $\left(G^{*}, q\right)$ of $G^{*}$.

Proof Since $\left|V\left(G^{*}\right)\right|=|V|+|P|$, we have $r\left(G^{*}, q\right) \leq 2(|V|+|P|)-3$ by Lemma 2.1. Thus we may assume that $\operatorname{def}(G) \geq 1$. Let $\mathcal{Q}=\left\{Q_{1}, Q_{2}, \ldots, Q_{t}\right\}$ be a tight partition of $V$. Since $\operatorname{def}(G) \geq 1$, we must have $t \geq 2$.

For $v \in V$, let $B^{*}(v)=\{v\} \cup E_{G}(v) \subset V\left(G^{*}\right)$, and let $X_{i}=\bigcup_{v \in Q_{i}} B^{*}(v)$ for $1 \leq i \leq t$. Then every edge of $G^{*}$ is induced by some $X_{i}$, and since $G$ has no isolated vertices, we have $\left|X_{i}\right| \geq 2$ for $1 \leq i \leq t$. Furthermore, $\sum_{i=1}^{t}\left|X_{i}\right|=|V|+|P|+$ $e_{G}(\mathcal{Q})$. Now we can use Lemma 2.1 to deduce that

$$
\begin{aligned}
r\left(G^{*}, q\right) & \leq \sum_{i=1}^{t}\left(2\left|X_{i}\right|-3\right)=2(|V|+|P|)+2 e_{G}(\mathcal{Q})-3 t \\
& =2(|V|+|P|)-3-\operatorname{def}(G) .
\end{aligned}
$$

We shall see later, as a corollary of our main result, that the equality can be attained in Lemma 3.2. (This corollary could also be proved directly using the characterization of the rank function of the rigidity matroid of a graph given by Lovász and Yemini [7].)

A multigraph $G$ is strong if $2 G$ has three edge-disjoint spanning trees. Equivalently, by Theorem 3.1(b), $G$ is strong if $\operatorname{def}(G)=0$, and, by Theorem $1.1, G$ is strong if $G$ can be realized as an infinitesimally rigid body-and-pin framework in $\mathbb{R}^{2}$. A subgraph $H$ of a multigraph $G$ is said to be a brick of $G$ if $H$ is a maximal strong subgraph of $G$. Thus bricks are induced subgraphs. We say that a multigraph $G=(V, E)$ is superstrong if $2 G-e$ has three edge-disjoint spanning trees for all $e \in E(2 G)$. Equivalently, by Theorem 3.1(b), $G$ is superstrong if $\operatorname{def}(G)=0$ and the only tight partition of $V$ is $\{V\}$ itself. A subgraph $H$ of $G$ is said to be a superbrick of $G$ if $H$ is a maximal superstrong subgraph of $G$. Thus superbricks are induced subgraphs.

The following properties of bricks, superbricks, and tight partitions were verified in [6] in a more general form.

Lemma 3.3 [6, Corollaries 2.5, 2.8] Let $G=(V, E)$ be a multigraph. Then the vertex sets of the bricks (resp. superbricks) of $G$ partition $V$.

The term brick partition (resp. superbrick partition) of $G$ refers to the partition of $V$ given by the vertex sets of the bricks (resp. superbricks) of $G$, see Fig. 4. We will also refer to the elements of the brick partition as bricks of $G$. It will be clear from the context whether we are referring to a subgraph or its vertex set. We shall frequently use the fact that the brick and superbrick partitions of $G$ are both tight. This follows from the next lemma.

Lemma 3.4 [6, Theorems 2.6, 2.9] Let $G=(V, E)$ be a multigraph and $\mathcal{P}$ be a tight partition of $V$. 

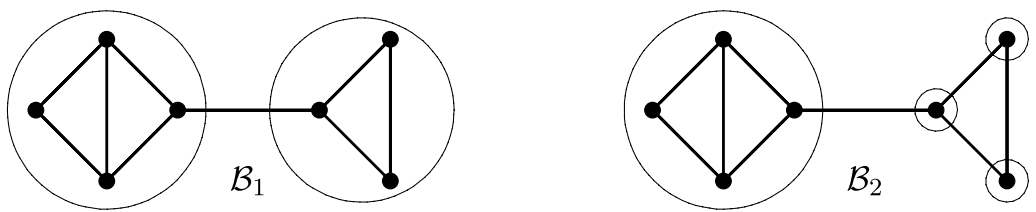

Fig. 4 The brick partition $\mathcal{B}_{1}$ and the superbrick partition $\mathcal{B}_{2}$ of the graph $G$ of Fig. 1. We have $\operatorname{def}(G)=\operatorname{def}_{G}\left(\mathcal{B}_{1}\right)=\operatorname{def}_{G}\left(\mathcal{B}_{2}\right)=1$

(a) If $\mathcal{P}$ is chosen so that $|\mathcal{P}|$ is as small as possible, then $\mathcal{P}$ is the brick partition of $G$.

(b) If $\mathcal{P}$ is chosen so that $|\mathcal{P}|$ is as large as possible, then $\mathcal{P}$ is the superbrick partition of $G$.

We need one further lemma from [6].

Lemma 3.5 [6, Lemma 2.3(a)] Let $\mathcal{P}$ be a tight partition of a multigraph $G$ and $X \in \mathcal{P}$. Then the subgraph of $G$ induced by $X$ is strong.

We also need a new lemma which follows from the fact that the brick partition of $G$ is tight.

Lemma 3.6 Let $G=(V, E)$ be a multigraph, and suppose that the subgraph of $G$ induced by $X$ is strong for some $X \subseteq V$. Then $\operatorname{def}(G)=\operatorname{def}(G / X)$, where $G / X$ denotes the multigraph obtained from $G$ by contracting $X$ into a single vertex.

\section{Pin-Line-Generic Body-and-Pin Realizations}

Let $G=(V, P)$ be a multigraph and $G^{*}$ be the body-and-pin graph of $G$. Consider a pin-collinear body-and-pin realization $\left(G^{*}, q\right)$ of $G$, and let $v \in V$. The line through $q(p), p \in E_{G}(v)$, is unique when $d_{G}(v) \geq 2$, and we denote it by $L_{\left(G^{*}, q\right)}(v)$. We extend this notation for vertices $v \in V$ with $d_{G}(v)=1$, say $E_{G}(v)=\{p\}$, by putting $L_{\left(G^{*}, q\right)}(v)$ equal to the line through $q(p)$ which is orthogonal to the line containing $q(v), q(p)$. We refer to the lines $L_{\left(G^{*}, q\right)}(v), v \in V$, as the pin-lines of $\left(G^{*}, q\right)$. Note that the infinitesimal rigidity of the subframework of $\left(G^{*}, q\right)$ induced by $\{v\} \cup E_{G}(v)$ implies that $q(v)$ cannot lie on $L_{\left(G^{*}, q\right)}(v)$ for each $v \in V$. Furthermore, if this condition holds, then $r\left(G^{*}, q\right)$ will be independent of the values chosen for $q(v)$ for all $v \in V$.

Let $\left(G^{*}, q\right)$ be a pin-collinear body-and-pin realization of a graph $G=(V, P)$ such that no pin-line of $\left(G^{*}, q\right)$ passes through the origin. Then, for each $v \in V$, there exists $a_{v}, b_{v} \in \mathbb{R}$ such that the pin-line of $v$ is given by

$$
L_{\left(G^{*}, q\right)}(v)=\left\{(x, y) \in \mathbb{R}^{2}: a_{v} x+b_{v} y=1\right\} .
$$

We say that $\left(G^{*}, q\right)$ is pin-line-generic if the (multi)set $\left\{a_{v}, b_{v}: v \in V\right\}$ is algebraically independent over $\mathbb{Q}$. Note that if $G$ contains two vertices $u$, $v$ which are 
joined by two or more edges in $G$, then we must necessarily have $L_{\left(G^{*}, q\right)}(u)=$ $L_{\left(G^{*}, q\right)}(v)$. Thus multigraphs cannot have pin-line-generic pin-collinear body-andpin realizations.

On the other hand, we shall see that every graph has a pin-line-generic pincollinear body-and-pin realization as a bar-and-joint framework. Furthermore, such a realization will maximize the rank over all pin-collinear body-and-pin realizations.

Lemma 4.1 Let $G=(V, P)$ be a graph. Then $r\left(G^{*}, q\right)$ achieves its maximum over all pin-collinear body-and-pin realizations of $G$ whenever $\left(G^{*}, q\right)$ is pin-linegeneric.

Proof Let $\left(G^{*}, q^{\prime}\right)$ be a pin-collinear body-and-pin realization of $G$ which maximizes $r\left(G^{*}, q\right)$. We may assume that no pin-line of $\left(G^{*}, q^{\prime}\right)$ passes through the origin. Let $L_{\left(G^{*}, q^{\prime}\right)}(v)=\left\{(x, y) \in \mathbb{R}^{2}: a_{v}^{\prime} x+b_{v}^{\prime} y=1\right\}$ for each $v \in V$. We show that, for any given $\epsilon>0$, there exists a pin-line-generic pin-collinear body-and-pin realization $\left(G^{*}, q\right)$ of $G$ with $\left\|q(u v)-q^{\prime}(u v)\right\|<\epsilon$ for all $u v \in P$. For any $\delta>0$ we may choose $\left(a_{v}, b_{v}\right)$ in $\mathbb{R}^{2}$ such that $\left\|\left(a_{v}, b_{v}\right)-\left(a_{v}^{\prime}, b_{v}^{\prime}\right)\right\|<\delta$ and such that the set $\left\{a_{v}, b_{v}: v \in V\right\}$ is algebraically independent over $\mathbb{Q}$. Let $L_{v}=\left\{(x, y) \in \mathbb{R}^{2}\right.$ : $\left.a_{v} x+b_{v} y=1\right\}$ for each $v \in V$. Define $q: V \cup P \rightarrow \mathbb{R}^{2}$ by putting $q(u v)$ equal to the point of intersection of $L_{u}$ and $L_{v}$ for each $u v \in P$ and putting $q(v)=(0,0)$ for all $v \in V$. Continuity implies that we will have $\left\|q(u v)-q^{\prime}(u v)\right\|<\epsilon$ if we make $\delta$ small enough. Lemma 2.2 now implies that $r\left(G^{*}, q\right)=r\left(G^{*}, q^{\prime}\right)$ for $\epsilon$ small enough, and hence $r\left(G^{*}, q\right)$ is also maximum.

Furthermore,

$$
q(u v)=d(u, v)^{-1}\left(b_{v}-b_{u}, a_{u}-a_{v}\right)
$$

where

$$
d(u, v)=\operatorname{det}\left(\begin{array}{ll}
a_{u} & b_{u} \\
a_{v} & b_{v}
\end{array}\right) .
$$

It follows that each entry in the rigidity matrix $R\left(G^{*}, q\right)$ is a rational function of $a_{v}, b_{v}, v \in V$. Thus $r\left(G^{*}, q\right)$ will be the same for all pin-line-generic realizations $\left(G^{*}, q\right)$.

\section{Maximum Rank of Pin-Collinear Body-and-Pin Realizations}

We now prove our main result, which determines the maximum rank of a pincollinear body-and-pin realization of a graph as a bar-and-joint framework. It will be extended to multigraphs at the end of this section.

Theorem 5.1 Let $G=(V, P)$ be a graph with no isolated vertices. Then the maximum rank of a pin-collinear body-and-pin realization of $G$ as a bar-and-joint framework is $2(|V|+|P|)-3-\operatorname{def}(G)$.

Proof By Lemma 3.2, it suffices to show that there exists a pin-collinear body-andpin realization $\left(G^{*}, q\right)$ of $G$ such that $r\left(G^{*}, q\right)=2(|V|+|P|)-3-\operatorname{def}(G)$. We 
proceed by contradiction. Suppose that there exists a graph $G=(V, P)$ such that, for all pin-collinear body-and-pin realizations $\left(G^{*}, q\right)$ of $G$, we have $r\left(G^{*}, q\right)<2(|V|+$ $|P|)-3-\operatorname{def}(G)$. We may suppose that $G$ has been chosen such that $|V|+|P|$ is as small as possible. It can easily be seen that $G$ has at least four vertices. We denote the number of vertices and edges of $G$ by $n$ and $m$, respectively. We will extend this notation using subscripts, so that for example, the number of vertices and edges in a graph $G_{1}$ will be denoted by $n_{1}$ and $m_{1}$, respectively. We will frequently use the fact that if $n_{1}+m_{1}<n+m$, then, by the minimality of $G$ and Lemma 4.1, there exists a pin-line-generic pin-collinear body-and-pin realization $\left(G_{1}^{*}, q\right)$ of $G_{1}$ with $r\left(G_{1}^{*}, q\right)=2\left(n_{1}+m_{1}\right)-3-\operatorname{def}\left(G_{1}\right)$. Since $\left(G_{1}^{*}, q\right)$ is pin-line-generic, no two pinlines of $\left(G_{1}^{*}, q\right)$ are parallel, and every point $q(p), p \in P_{1}$, belongs to exactly two pin-lines in $\left(G_{1}^{*}, q\right)$. We next prove eight claims which establish structural properties of our minimal counterexample $G$.

\section{Claim 5.2 $G$ is connected.}

Proof Suppose that the claim is false. Then there exist disjoint subgraphs $G_{1}, G_{2}$ of $G$ such that $G=G_{1} \cup G_{2}$. Since the brick partition of $G$ is just the union of the brick partitions of $G_{1}$ and $G_{2}$, we may use Lemma 3.4(a) to deduce that $\operatorname{def}(G)=$ $\operatorname{def}\left(G_{1}\right)+\operatorname{def}\left(G_{2}\right)+3$. By the minimality of $G$, there exists a pin-collinear bodyand-pin realization $\left(G_{i}^{*}, q_{i}\right)$ of $G_{i}$ such that $r\left(G_{i}^{*}, q_{i}\right)=2\left(n_{i}+m_{i}\right)-3-\operatorname{def}\left(G_{i}\right)$ for each $i \in\{1,2\}$. Taking the union of $\left(G_{1}^{*}, q_{1}\right)$ and $\left(G_{2}^{*}, q_{2}\right)$, we obtain a pin-collinear body-and-pin realization $\left(G^{*}, q\right)$ of $G$. By Lemma 2.6

$$
\begin{aligned}
r\left(G^{*}, q\right) & =r\left(G_{1}^{*}, q_{1}\right)+r\left(G_{2}^{*}, q_{2}\right) \\
& =2\left(n_{1}+m_{1}\right)-3-\operatorname{def}\left(G_{1}\right)+2\left(n_{2}+m_{2}\right)-3-\operatorname{def}\left(G_{2}\right) \\
& =2(n+m)-3-\operatorname{def}(G) .
\end{aligned}
$$

This contradicts the choice of $G$.

Claim 5.3 For each $v \in V, d_{G}(v) \geq 2$.

Proof Suppose that there exists $v_{1} \in V$ with $d_{G}\left(v_{1}\right)=1$. Let $p_{1}=u_{1} v_{1}$ be the edge of $G$ incident to $v_{1}$ and $G_{1}=G-v_{1}$. Let $\mathcal{Q}_{1}$ be a tight partition of $G_{1}$. Put $B=\left\{v_{1}\right\}$ and let $\mathcal{Q}=\mathcal{Q}_{1} \cup\{B\}$. Then $\operatorname{def}(G) \geq \operatorname{def}_{G}(\mathcal{Q})=\operatorname{def}_{G_{1}}\left(\mathcal{Q}_{1}\right)+1=\operatorname{def}\left(G_{1}\right)+1$. By the minimality of $G$ and Lemma 4.1, there exists a pin-line-generic pin-collinear body-and-pin realization $\left(G_{1}^{*}, q_{1}\right)$ of $G_{1}$ such that $r\left(G_{1}^{*}, q_{1}\right)=2\left(n_{1}+m_{1}\right)-3-$ $\operatorname{def}\left(G_{1}\right)$. Choose $p_{2} \in E_{G_{1}}\left(u_{1}\right)$. Let $Q_{1}$ be a point on $L_{\left(G_{1}^{*}, q_{1}\right)}\left(u_{1}\right)$ such that $Q_{1} \neq$ $q_{1}(p)$ for all $p \in E_{G_{1}}\left(u_{1}\right)$. Choose another point $Q_{2} \neq Q_{1}$. We may now extend $\left(G_{1}^{*}, q_{1}\right)$ to a pin-collinear body-and-pin realization $\left(G^{*}, q\right)$ of $G$ by putting $q\left(p_{1}\right)=$ $Q_{1}$ and $q\left(v_{1}\right)=Q_{2}$. Since $G^{*}$ can be obtained from $G_{1}^{*}$ by performing a 0 -extension (adding the vertex $p_{1}$ and edges $p_{1} u_{1}, p_{1} p_{2}$ ), adding the vertex $v_{1}$ and edge $v_{1} p_{1}$ and finally adding the remaining edges of $E\left(G^{*}\right)$, Lemma 2.3 implies that

$r\left(G^{*}, q\right) \geq r\left(G_{1}^{*}, q_{1}\right)+3=2\left(n_{1}+m_{1}\right)-3-\operatorname{def}\left(G_{1}\right)+3 \geq 2(n+m)-3-\operatorname{def}(G)$.

This contradicts the choice of $G$. 


\section{Claim $5.4 G$ is 2-edge-connected.}

Proof Suppose that the claim is false. Then there exist $p_{0} \in P$ and disjoint subgraphs $G_{1}, G_{2}$ of $G_{0}=G-p_{0}$ such that $G_{0}=G_{1} \cup G_{2}$. By the minimality of $G$ and Lemma 4.1, there exists a pin-line-generic pin-collinear body-and-pin realization $\left(G_{0}^{*}, q_{0}\right)$ of $G_{0}$ which satisfies $r\left(G_{0}^{*}, q_{0}\right)=2\left(n_{0}+m_{0}\right)-3-\operatorname{def}\left(G_{0}\right)$. Since the brick partition of $G$ is just the union of the brick partitions of $G_{1}$ and $G_{2}$, we may use Lemma 3.4(a) to deduce that $\operatorname{def}(G)=\operatorname{def}\left(G_{0}\right)-2$. Let $u_{i}$ be the vertex of $G_{i}$ incident to $p_{0}$ for each $i \in\{1,2\}$ and $Q$ be the point of intersection of the lines $L_{\left(G_{0}^{*}, q_{0}\right)}\left(u_{1}\right)$ and $L_{\left(G_{0}^{*}, q_{0}\right)}\left(u_{2}\right)$. We may extend $\left(G_{0}^{*}, q_{0}\right)$ to a pin-collinear body-andpin realization $\left(G^{*}, q\right)$ of $G$ by putting $q\left(p_{0}\right)=Q$. Let $H_{i}$ be the subgraph of $G^{*}$ induced by $V\left(G_{i}^{*}\right) \cup\left\{p_{0}\right\}, q_{i}$ the restriction of $q$ to $V\left(G_{i}^{*}\right)$ and $q_{i}^{\prime}$ the restriction of $q$ to $V\left(H_{i}\right)$ for $i \in\{1,2\}$. Lemmas 2.3 and 2.6 imply that

$$
\begin{aligned}
r\left(G^{*}, q\right) & =r\left(H_{1}, q_{1}^{\prime}\right)+r\left(H_{2}, q_{2}^{\prime}\right) \geq r\left(G_{1}^{*}, q_{1}\right)+2+r\left(G_{2}^{*}, q_{2}\right)+2 \\
& \geq r\left(G_{0}^{*}, q_{0}\right)+4=2\left(n_{0}+m_{0}\right)-3-\operatorname{def}\left(G_{0}\right)+4 \\
& =2(n+m)-3-\operatorname{def}(G) .
\end{aligned}
$$

This contradicts the choice of $G$.

Claim 5.5 For each $v \in V, d_{G}(v) \geq 3$.

Proof Suppose that there exists $v_{1} \in V$ with $d_{G}\left(v_{1}\right)=2$. Let $p_{1}=u_{1} v_{1}$ and $p_{2}=$ $u_{2} v_{1}$ be the edges of $G$ incident to $v_{1}$ and $G_{1}=G-v_{1}$. Let $\mathcal{B}_{1}$ be the brick partition of $G_{1}$.

We first consider the case where $u_{1}$ and $u_{2}$ both belong to the same brick $B_{1}$ of $G_{1}$. Let $B=B_{1}+v_{1}$ and $\mathcal{Q}=\mathcal{B}_{1}-\left\{B_{1}\right\} \cup\{B\}$. Then

$$
\operatorname{def}(G) \geq \operatorname{def}_{G}(\mathcal{Q})=\operatorname{def}_{G_{1}}\left(\mathcal{B}_{1}\right)=\operatorname{def}\left(G_{1}\right) .
$$

By the minimality of $G$ and Lemma 4.1, there exists a pin-line-generic pin-collinear body-and-pin realization $\left(G_{1}^{*}, q_{1}\right)$ of $G_{1}$ such that

$$
r\left(G_{1}^{*}, q_{1}\right)=2\left(n_{1}+m_{1}\right)-3-\operatorname{def}\left(G_{1}\right) \geq 2(n+m)-9-\operatorname{def}(G) .
$$

For $i \in\{1,2\}$, let $Q_{i}$ be a point on $L_{\left(G_{1}^{*}, q_{1}\right)}\left(u_{i}\right)$ such that $Q_{i} \neq q_{1}(p), p \in E_{G_{1}}\left(u_{i}\right)$, and $Q_{1} \neq Q_{2}$. Choose a point $Q$ which does not lie on the line through $Q_{1}, Q_{2}$. We may now extend $\left(G_{1}^{*}, q_{1}\right)$ to a pin-collinear body-and-pin realization $\left(G^{*}, q\right)$ of $G$ by putting $q\left(p_{i}\right)=Q_{i}$ for $i \in\{1,2\}$ and $q\left(v_{1}\right)=Q$. Lemma 2.3 implies that $r\left(G^{*}, q\right) \geq$ $r\left(G_{1}^{*}, q_{1}\right)+6 \geq 2(n+m)-3-\operatorname{def}(G)$. This contradicts the choice of $G$.

Thus $u_{1}$ and $u_{2}$ must belong to distinct bricks of $G_{1}$. Since the bricks of $G_{1}$ are the maximal strong subgraphs of $G_{1}$, this implies that

$$
\text { no strong subgraph of } G_{1} \text { can contain both } u_{1} \text { and } u_{2} \text {. }
$$

Let $B=\left\{v_{1}\right\}$ and $\mathcal{Q}=\mathcal{B}_{1} \cup\{B\}$. Then

$$
\operatorname{def}(G) \geq \operatorname{def}_{G}(\mathcal{Q})=\operatorname{def}_{G_{1}}\left(\mathcal{B}_{1}\right)-1=\operatorname{def}\left(G_{1}\right)-1 .
$$


Consider the following three cases.

Case 1. $u_{1} u_{2} \notin P \quad$ Let $p_{0}=u_{1} u_{2}$ and $G_{2}=G_{1}+p_{0}$. If $\operatorname{def}\left(G_{2}\right)=\operatorname{def}\left(G_{1}\right)$, then $u_{1}, u_{2}$ would belong to the same set in some tight partition of $G_{1}$. Since each set in a tight partition induces a strong subgraph of $G_{1}$ by Lemma 3.5, this would contradict (2). Hence $\operatorname{def}\left(G_{2}\right) \leq \operatorname{def}\left(G_{1}\right)-1$. By the minimality of $G$ and Lemma 4.1, there exists a pin-line-generic pin-collinear body-and-pin realization $\left(G_{2}^{*}, q_{2}\right)$ of $G_{2}$ such that

$$
r\left(G_{2}^{*}, q_{2}\right)=2\left(n_{2}+m_{2}\right)-3-\operatorname{def}\left(G_{2}\right) \geq 2\left(n_{1}+m_{1}\right)-3-\operatorname{def}\left(G_{1}\right)+3 .
$$

Let $q_{1}$ be the restriction of $q_{2}$ to $V\left(G_{1}^{*}\right)$. Then $\left(G_{1}^{*}, q_{1}\right)$ is a pin-line-generic pin-collinear body-and-pin realization of $G_{1}$, so, again by the minimality of $G$ and Lemma 4.1, $r\left(G_{1}^{*}, q_{1}\right)=2\left(n_{1}+m_{1}\right)-3-\operatorname{def}\left(G_{1}\right)$. Since $G_{1}^{*}+p_{0}+p_{0} u_{1}+p_{0} u_{2}$ is a 0 -extension of $G_{1}^{*}$, Lemma 2.3 implies that $r\left(G_{1}^{*}+p_{0}+p_{0} u_{1}+p_{0} u_{2}, q_{2}\right)=$ $r\left(G_{1}^{*}, q_{1}\right)+2$. Since $\left(G_{1}^{*}, q_{1}\right)$ is a subframework of $\left(G_{2}^{*}, q_{2}\right)$, we may use (3) to deduce that

$$
r\left(G_{1}^{*}+p_{0}+p_{0} u_{1}+p_{0} u_{2}+p_{0} p_{4}, q_{2}\right)=2\left(n_{1}+m_{1}\right)-3-\operatorname{def}\left(G_{1}\right)+3
$$

for some $p_{4} \in E_{G_{1}}\left(u_{1}\right) \cup E_{G_{1}}\left(u_{2}\right)$. By symmetry we may assume that $p_{4} \in E_{G_{1}}\left(u_{1}\right)$. Let $H_{2}=G_{1}^{*}+p_{0}+p_{0} u_{1}+p_{0} u_{2}+p_{0} p_{4}$. By Lemma 2.2, $r\left(H_{2}, q_{2}\right)$ does not decrease if we move $q_{2}\left(p_{0}\right)$ in a small enough neighbourhood to a new position $Q_{1}$ in such a way that it remains on $L_{\left(G_{2}^{*}, q_{2}\right)}\left(u_{1}\right)$ but no longer lies on $L_{\left(G_{2}^{*}, q_{2}\right)}\left(u_{2}\right)$ and such that the line $L_{0}$ through $Q_{1}$ and $q_{2}\left(u_{2}\right)$ intersects $L_{\left(G_{2}^{*}, q_{2}\right)}\left(u_{2}\right)$ at a point $Q_{2} \neq q_{2}(p)$ for all $p \in E_{G_{2}}\left(u_{2}\right)$. Choose a point $Q_{0}$ such that $Q_{0}, Q_{1}, Q_{2}$ are not collinear. Define $q: V\left(G^{*}\right) \rightarrow \mathbb{R}^{2}$ by putting $q(x)=q_{2}(x)$ for $x \in V\left(G_{2}^{*}\right)-p_{0}, q\left(p_{1}\right)=Q_{1}$, $q\left(p_{2}\right)=Q_{2}$, and $q\left(v_{1}\right)=Q_{0}$. Then $\left(G^{*}, q\right)$ is a pin-collinear body-and-pin realization of $G$. Since $G^{*}$ can be obtained from $H_{2}$ by first relabelling $p_{0}$ as $p_{1}$, then performing a 1-extension (deleting $p_{1} u_{2}$ and adding $p_{2}, p_{2} p_{1}, p_{2} u_{2}, p_{2} p_{3}$ for some $p_{3} \in E_{G_{1}}\left(u_{2}\right)$ ), then performing a 0 -extension (adding $v_{1}, v_{1} p_{1}, v_{1} p_{2}$ ), and finally adding the remaining edges of $E\left(G^{*}\right)$, Lemmas 2.3 and 2.4 imply that

$r\left(G^{*}, q\right) \geq r\left(H_{2}, q_{2}\right)+4 \geq 2\left(n_{1}+m_{1}\right)-3-\operatorname{def}\left(G_{1}\right)+7 \geq 2(n+m)-3-\operatorname{def}(G)$.

This contradicts the choice of $G$.

Case 2. $u_{1} u_{2} \in P$ and $d_{G}\left(u_{i}\right) \geq 3$ for all $i \in\{1,2\} \quad$ Let $p_{3}=u_{1} u_{2}, E_{G_{1}}\left(u_{1}\right)=$ $\left\{p_{3}, p_{4}, \ldots, p_{j}\right\}$ and $E_{G_{1}}\left(u_{2}\right)=\left\{p_{3}, p_{j+1}, p_{j+2}, \ldots, p_{k}\right\}$. Since $d_{G}\left(u_{1}\right), d_{G}\left(u_{2}\right) \geq 3$, $j \geq 4$ and $k \geq 5$. Since $u_{1} u_{2} \in P$ and $K_{3}$ is strong, we may use (2) to deduce that no vertex of $G_{1}$ is adjacent to both $u_{1}$ and $u_{2}$. Thus the (multi)graph $G_{3}$ obtained from $G_{1}$ by contracting the edge $p_{3}$ onto a new vertex $z$ contains no parallel edges. Since $d_{G_{1}}\left(u_{1}\right) \geq 2$ and $d_{G_{1}}\left(u_{2}\right) \geq 2$, we have $d_{G_{3}}(z) \geq 2$, and hence $G_{3}$ contains no isolated vertices.

Suppose that $\operatorname{def}\left(G_{3}\right) \geq \operatorname{def}\left(G_{1}\right)$. Let $\mathcal{B}_{3}$ be the brick partition of $G_{3}$ and choose $B_{3} \in \mathcal{B}_{3}$ with $z \in B_{3}$. Let $B_{3}^{\prime}=\left(B_{3}-\{z\}\right) \cup\left\{u_{1}, u_{2}\right\}$ and $\mathcal{Q}_{1}=\left(\mathcal{B}_{3}-B_{3}\right)+B_{3}^{\prime}$. Then

$$
\operatorname{def}_{G_{1}}\left(\mathcal{Q}_{1}\right)=\operatorname{def}_{G_{3}}\left(\mathcal{B}_{3}\right)=\operatorname{def}\left(G_{3}\right) \geq \operatorname{def}\left(G_{1}\right),
$$



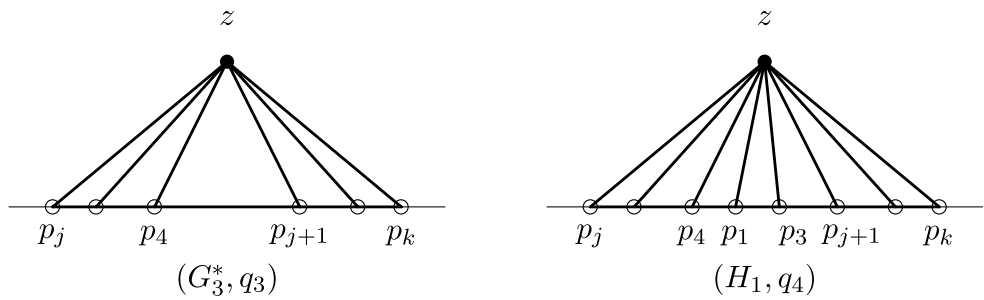

Fig. 5 The frameworks $\left(G_{3}^{*}, q_{3}\right)$ and $\left(H_{1}, q_{4}\right)$ in the proof of Case 2 of Claim 5.5

and hence $\mathcal{Q}_{1}$ is a tight partition of $G_{1}$. Each brick of $G_{1}$ which does not contain $u_{1}$ or $u_{2}$ is contained in a brick of $G_{3}$. Furthermore, if $X_{1}$ and $X_{2}$ are the distinct bricks of $G_{1}$ containing $u_{1}$ and $u_{2}$ respectively, then $X_{1}-u_{1}, X_{2}-u_{2}$ and $z$ are all contained in the same brick of $G_{3}$. Thus the number of bricks of $G_{1}$ is strictly greater than $\left|\mathcal{B}_{3}\right|=\left|\mathcal{Q}_{1}\right|$. Now $\mathcal{Q}_{1}$ contradicts the fact that the brick partition of $G_{1}$ is the tight partition with the fewest parts. Thus $\operatorname{def}\left(G_{3}\right) \leq \operatorname{def}\left(G_{1}\right)-1$.

By the minimality of $G$ and Lemma 4.1 , there exists a pin-line-generic pincollinear body-and-pin realization $\left(G_{3}^{*}, q_{3}\right)$ of $G_{3}$ such that

$$
r\left(G_{3}^{*}, q_{3}\right)=2\left(n_{3}+m_{3}\right)-3-\operatorname{def}\left(G_{3}\right) .
$$

Each vertex $p_{i}, 4 \leq i \leq k$, lies on exactly two pin-lines of $\left(G_{3}^{*}, q_{3}\right)$. Let $L_{i}$ be the pin line of $\left(G_{3}^{*}, q_{3}\right)$ which contains $p_{i}$ and is distinct from $L_{\left(G_{3}^{*}, q_{3}\right)}(z)$.

Let $S=\left\{p_{s} p_{t}: 4 \leq s \leq j, j+1 \leq t \leq k,(s, t) \neq(4, j+1)\right\}, H_{0}=G_{3}^{*}-S$, $F$ be the subgraph of $H_{0}$ induced by $\{z\} \cup\left\{p_{i}: 4 \leq i \leq k\right\}$, and $q_{0}$ be the restriction of $q_{3}$ to $F$. Since $\left(F, q_{0}\right)$ is infinitesimally rigid, $r\left(H_{0}, q_{3}\right)=r\left(G_{3}^{*}, q_{3}\right)$. Let $H_{1}$ be the graph obtained from $H_{0}-p_{4} p_{j+1}$ by adding two new vertices, $p_{1}, p_{3}$, and edges $p_{1} p_{3}, p_{1} p_{4}, p_{1} z, p_{3} p_{j+1}, p_{3} z$, see Fig. 5. Choose two distinct points $Q_{1}, Q_{3}$ on $L_{\left(G_{3}^{*}, q_{3}\right)}(z)$ such that $Q_{i} \neq q_{3}(p)$ for all $i \in\{1,3\}$ and $p \in E_{G_{3}}(z)$, and define $q_{4}: V\left(H_{1}\right) \rightarrow \mathbb{R}^{2}$ by $q_{4}(x)=q_{3}(x)$ for $x \in V\left(G_{3}^{*}\right), q_{4}\left(p_{1}\right)=Q_{1}$ and $q_{4}\left(p_{3}\right)=$ $Q_{3}$. Since $\left(H_{1}, q_{4}\right)$ can be obtained from $\left(H_{0}, q_{3}\right)$ by applying two 1-extensions, Lemma 2.4 implies that $r\left(H_{1}, q_{4}\right)=r\left(H_{0}, q_{3}\right)+4=r\left(G_{3}^{*}, q_{3}\right)+4$.

Let $H_{2}$ be the graph obtained from $H_{1}-z$ by adding two new vertices, $u_{1}, u_{2}$, edges $u_{1} p_{i}$ for $i \in\{1,3,4, \ldots, j\}$, and edges $u_{2} p_{i}$ for $i \in\{1,3, j+1, j+2, \ldots, k\}$. Define $q_{5}: V\left(H_{2}\right) \rightarrow \mathbb{R}^{2}$ by $q_{5}(x)=q_{4}(x)$ for $x \in V\left(H_{1}\right)-z$ and $q_{5}\left(u_{1}\right)=q_{5}\left(u_{2}\right)=$ $q_{4}(z)$. Since $\left(H_{2}, q_{5}\right)$ can be obtained from $\left(H_{1}, q_{4}\right)$ by a vertex-split, Lemma 2.5 implies that $r\left(H_{2}, q_{5}\right) \geq r\left(H_{1}, q_{4}\right)+2$. By Lemma 2.2, there exists a neighbourhood $S_{i}$ around each point $q_{5}\left(p_{i}\right), i \in\{1,4, \ldots, j\}$, such that $r\left(H_{2}, q_{5}\right)$ does not decrease if we move $q_{5}\left(p_{i}\right)$ within $S_{i}$. Thus we may modify $\left(H_{2}, q_{5}\right)$ by moving each point $q_{5}\left(p_{i}\right), i \in\{1,4, \ldots, j\}$, slightly, in such a way that it continues to lie on $L_{i}$ and belong to $S_{i}$, and also such that $p_{1}, p_{3}, p_{4}, \ldots, p_{j}$ all lie on a line $L_{0}$ which is not parallel to $L_{\left(G_{3}^{*}, q_{3}\right)}(z)$. Let $\left(H_{2}, \tilde{q}_{5}\right)$ be the modified framework, see Fig. 6. (We may imagine $L_{0}$ is obtained by a small rotation of $L_{\left(G_{3}^{*}, q_{3}\right)}(z)$ about the point $q_{5}\left(p_{3}\right)$.)

Let $L_{1}$ be the line through $\tilde{q}_{5}\left(p_{1}\right)$ and $\tilde{q}_{5}\left(u_{2}\right)$. By using Lemma 2.2 to move $\tilde{q}_{5}\left(u_{2}\right)$ if necessary, we may suppose that $Q_{2}$, the point of intersection of $L_{1}$ and $L_{\left(G_{3}^{*}, q_{3}\right)}(z)$, is distinct from $\tilde{q}_{5}(p)$ for all $p \in E_{G_{1}}\left(u_{2}\right)$. Choose a point $Q_{0}$ which does not lie on 


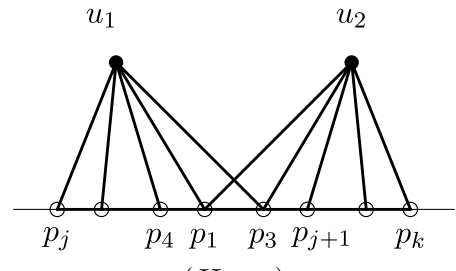

$\left(H_{2}, q_{5}\right)$

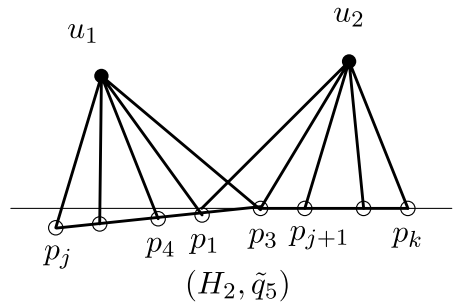

$\left(H_{2}, \tilde{q}_{5}\right)$

Fig. 6 The frameworks $\left(H_{2}, q_{5}\right)$ and $\left(H_{2}, \tilde{q}_{5}\right)$ in the proof of Case 2 of Claim 5.5

$L_{1}$. Define $q: V\left(G^{*}\right) \rightarrow \mathbb{R}^{2}$ by $q(x)=\tilde{q}_{5}(x)$ for $x \in V\left(G^{*}\right)-\left\{p_{2}, v_{1}\right\}, q\left(p_{2}\right)=$ $Q_{2}$ and $q\left(v_{1}\right)=Q_{0}$. Then $\left(G^{*}, q\right)$ is a pin-collinear body-and-pin realization of $G$. Since $\left(G^{*}, q\right)$ can be obtained from $\left(H_{2}, \tilde{q}_{5}\right)$ by a 1-extension (which deletes $u_{2} p_{1}$ and adds a new vertex $p_{2}$ and edges $p_{2} p_{1}, u_{2} p_{2}, p_{2} p_{3}$ ), a 0 -extension (which adds $v_{1}, v_{1} p_{1}, v_{1} p_{2}$ ) and by adding other edges, we have

$$
\begin{aligned}
r\left(G^{*}, q\right) & \geq r\left(H_{2}, \tilde{q}_{5}\right)+4 \geq r\left(H_{1}, q_{4}\right)+6=r\left(G_{3}^{*}, q_{3}\right)+10 \\
& =2\left(n_{3}+m_{3}\right)-3-\operatorname{def}\left(G_{3}\right)+10 \geq 2(n+m)-3-\operatorname{def}(G),
\end{aligned}
$$

since $\operatorname{def}(G) \geq \operatorname{def}\left(G_{1}\right)-1 \geq \operatorname{def}\left(G_{3}\right)$. This contradicts the choice of $G$.

Case 3. $u_{1} u_{2} \in P$ and $d_{G}\left(u_{i}\right)=2$ for some $i \in\{1,2\} \quad$ Let $p_{3}=u_{1} u_{2}$. Suppose, without loss of generality, that $E_{G}\left(u_{2}\right)=\left\{p_{2}, p_{3}\right\}$. Let $G_{4}=G-\left\{v_{1}, u_{2}\right\}$. Note that $G$ is obtained from $G_{4}$ by attaching a complete graph on three vertices (which is strong) at vertex $u_{1}$, and hence $\operatorname{def}\left(G_{4}\right)=\operatorname{def}(G)$. By Claim 5.4 we have $\left|E_{G_{4}}\left(u_{1}\right)\right| \geq 2$.

By the minimality of $G$ and Lemma 4.1 , there exists a pin-line-generic pincollinear body-and-pin realization $\left(G_{4}^{*}, q_{6}\right)$ of $G_{4}$ such that

$$
r\left(G_{4}^{*}, q_{6}\right)=2\left(n_{4}+m_{4}\right)-3-\operatorname{def}\left(G_{4}\right) .
$$

Let $p_{4} \in E_{G_{4}}\left(u_{1}\right)$. Let $H_{4}$ be the graph obtained from $G_{4}^{*}$ by adding new vertices $p_{1}, p_{2}, p_{3}, v_{1}, u_{2}$ and edges $p_{1} u_{1}, p_{1} p_{4}, p_{3} u_{1}, p_{3} p_{4}, p_{2} p_{1}, p_{2} p_{3}, v_{1} p_{1}, v_{1} p_{2}, u_{2} p_{3}$ and $u_{2} p_{2}$. Choose distinct points $Q_{1}, Q_{3}$ on $L_{\left(G_{4}^{*}, q_{6}\right)}\left(u_{1}\right)$ such that $Q_{i} \neq q_{6}(p)$ for all $i \in\{1,3\}$ and $p \in E_{G_{4}}\left(u_{1}\right)$. Choose a point $Q_{2}$ which is not on $L_{\left(G_{4}^{*}, q_{6}\right)}\left(u_{1}\right)$ and choose points $Q_{5}, Q_{6}$ such that $Q_{5}$ is not on the line through $Q_{1}, Q_{2}$, and $Q_{6}$ is not on the line through $Q_{3}, Q_{2}$. Define $q_{7}: V\left(H_{4}\right) \rightarrow \mathbb{R}^{2}$ by $q_{7}(x)=q_{6}(x)$ for $x \in V\left(G_{4}^{*}\right), q_{7}\left(p_{1}\right)=Q_{1}, q_{7}\left(p_{3}\right)=Q_{3}, q_{7}\left(p_{2}\right)=Q_{2}, q_{7}\left(v_{1}\right)=Q_{5}$, and $q_{7}\left(u_{2}\right)=$ $Q_{6}$, see Fig. 7. Since $\left(H_{4}, q_{7}\right)$ can be obtained from $\left(G_{4}^{*}, q_{6}\right)$ by five 0 -extensions, Lemma 2.3 implies that $r\left(H_{4}, q_{7}\right)=r\left(G_{4}^{*}, q_{6}\right)+10$. Since $\operatorname{def}\left(G_{4}\right)=\operatorname{def}(G)$ and $\left|V\left(G^{*}\right)\right|=\left|V\left(G_{4}^{*}\right)\right|+5$, we have

$$
r\left(G^{*}, q_{7}\right) \geq r\left(H_{4}, q_{7}\right)=r\left(G_{4}^{*}, q_{6}\right)+10=2(n+m)-3-\operatorname{def}(G) .
$$

This contradicts the choice of $G$. 


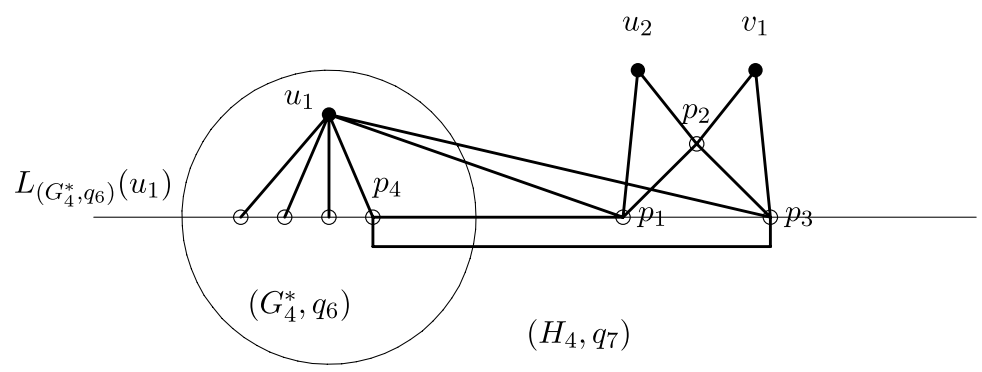

Fig. 7 The framework $\left(H_{4}, q_{7}\right)$ in the proof of Case 3 of Claim 5.5

Claim 5.6 For each $p \in P, \operatorname{def}(G-p) \geq \operatorname{def}(G)+1$.

Proof Clearly $\operatorname{def}(G-p) \geq \operatorname{def}(G)$. Suppose that $\operatorname{def}\left(G-p_{1}\right)=\operatorname{def}(G)$ for some $p_{1} \in P$. Let $p_{1}=v_{1} v_{2}$ and $G_{1}=G-p_{1}$. By the minimality of $G$ and Lemma 4.1, there exists a pin-line-generic pin-collinear body-and-pin realization $\left(G_{1}^{*}, q_{1}\right)$ of $G_{1}$ such that $r\left(G_{1}^{*}, q_{1}\right)=2\left(n_{1}+m_{1}\right)-3-\operatorname{def}\left(G_{1}\right)$. Let $Q$ be the point of intersection of $L_{\left(G_{1}^{*}, q_{1}\right)}\left(v_{1}\right)$ and $L_{\left(G_{1}^{*}, q_{1}\right)}\left(v_{2}\right)$. We may extend $\left(G_{1}^{*}, q_{1}\right)$ to a pin-collinear bodyand-pin realization $\left(G^{*}, q\right)$ of $G$ by putting $q\left(p_{1}\right)=Q$. Lemma 2.3 implies that

$r\left(G^{*}, q\right) \geq r\left(G_{1}^{*}, q_{1}\right)+2=2\left(n_{1}+m_{1}\right)-3-\operatorname{def}\left(G_{1}\right)+2=2(n+m)-3-\operatorname{def}(G)$.

This contradicts the choice of $G$.

Claim 5.7 G is not 3-edge-connected.

Proof Choose $p_{0} \in P$ and let $G_{0}=G-p_{0}$. By Claim 5.6, $\operatorname{def}\left(G_{0}\right) \geq \operatorname{def}(G)+1 \geq 1$. Thus $G_{0}$ is not strong. Let $\mathcal{B}_{0}$ be the brick partition of $G_{0}$. We have $\operatorname{def}\left(G_{0}\right)=$ $\operatorname{def}_{G_{0}}\left(\mathcal{B}_{0}\right)=3\left(\left|\mathcal{B}_{0}\right|-1\right)-2 e_{G_{0}}\left(\mathcal{B}_{0}\right) \geq 1$. Thus $2 e_{G_{0}}\left(\mathcal{B}_{0}\right) \leq 3\left|\mathcal{B}_{0}\right|-4$ and $2 e_{G}\left(\mathcal{B}_{0}\right) \leq$ $3\left|\mathcal{B}_{0}\right|-2$. Hence, there exists $B \in \mathcal{B}_{0}$ such that $d_{G}(B) \leq 2$.

Claim 5.8 If $S=\left\{p_{1}, p_{2}\right\}$ is a 2-edge-cut of $G$, then $\operatorname{def}\left(G-p_{1}\right)=\operatorname{def}(G)+2$.

Proof Let $p_{1}=u v$ and $G_{1}=G-p_{1}$. It follows from the definition of $\operatorname{def}(G)$ and Claim 5.6 that $\operatorname{def}(G)+1 \leq \operatorname{def}\left(G_{1}\right) \leq \operatorname{def}(G)+2$. Suppose that $\operatorname{def}\left(G_{1}\right)=$ $\operatorname{def}(G)+1$. By the minimality of $G$ and Lemma 4.1, there exists a pin-line-generic pin-collinear body-and-pin realization $\left(G_{1}^{*}, q_{1}\right)$ of $G_{1}$ such that $r\left(G_{1}^{*}, q_{1}\right)=2\left(n_{1}+\right.$ $\left.m_{1}\right)-3-\operatorname{def}\left(G_{1}\right)$. Let $Q$ be the point of intersection of $L_{\left(G_{1}^{*}, q_{1}\right)}(u)$ and $L_{\left(G_{1}^{*}, q_{1}\right)}(v)$. We may assume that $Q$ does not lie on the line through $q_{1}\left(p_{2}\right)$ and $q_{1}(v)$, since if it does, then we can use Lemma 2.2 to move $q_{1}(v)$ in a small neighbourhood without decreasing $r\left(G_{1}^{*}, q_{1}\right)$. Choose $p_{3} \in E_{G_{1}}(u)$ and let $H_{1}$ be the graph obtained from $G_{1}^{*}$ by adding the vertex $p_{1}$ and edges $p_{1} u, p_{1} p_{3}$. We may extend $\left(G_{1}^{*}, q_{1}\right)$ to a bar-and-joint realization $\left(H_{1}, q\right)$ of $H_{1}$ by putting $q\left(p_{1}\right)=Q$. Lemma 2.3 implies that $r\left(H_{1}, q\right)=r\left(G_{1}^{*}, q_{1}\right)+2$. Let $H_{2}$ be obtained from $H_{1}$ by adding the edge $p_{1} v$. Since $Q$ is not on the line through $q_{1}\left(p_{2}\right)$ and $q_{1}(v)$, the infinitesimal motion of $\left(H_{1}, q\right)$ which keeps both $p_{2}$ and the component of $H_{1}-p_{2}$ containing $u$ fixed and 
Fig. 8 The graphs $G_{1}$ and $G_{2}$
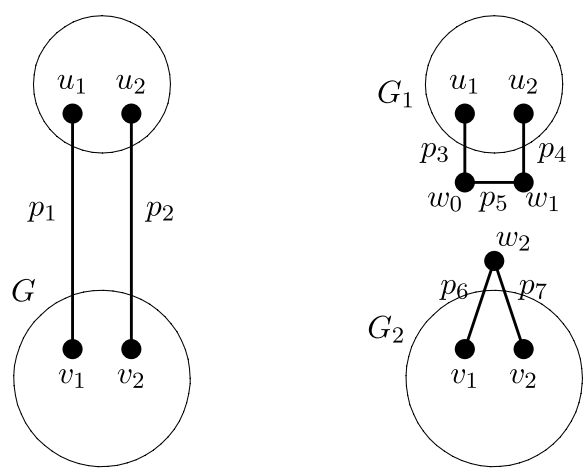

rotates the other component about $p_{2}$ is not an infinitesimal motion of $\left(\mathrm{H}_{2}, q\right)$ (since it is not orthogonal to $\left.q\left(p_{1}\right)-q(v)\right)$. The degree of freedom of $\left(H_{2}, q\right)$ is less than $\left(H_{1}, q\right)$ and $r\left(H_{2}, q\right) \geq r\left(H_{1}, q\right)+1$. Hence $\left(G^{*}, q\right)$ is a pin-collinear body-and-pin realization of $G$ for which

$$
\begin{aligned}
r\left(G^{*}, q\right) & \geq r\left(H_{2}, q\right) \geq r\left(G_{1}^{*}, q_{1}\right)+3 \\
& =2\left(n_{1}+m_{1}\right)-3-\operatorname{def}\left(G_{1}\right)+3=2(n+m)-3-\operatorname{def}(G) .
\end{aligned}
$$

This contradicts the choice of $G$.

Claim 5.9 $G$ is not superstrong.

Proof Suppose that $G$ is superstrong. Then $2 e_{G}(\mathcal{P}) \geq 3(|\mathcal{P}|-1)+1$ for all partitions $\mathcal{P}$ of $G$ with $|\mathcal{P}| \geq 2$. Hence $\operatorname{def}(G-p) \leq \operatorname{def}(G)+1$ for all $p \in P$. This contradicts Claims 5.7 and 5.8.

We now continue the proof of the theorem. Let $\mathcal{B}$ be the superbrick partition of $G$. By Claim 5.9, $|\mathcal{B}| \geq 2$. Since $\operatorname{def}(G)=3(|\mathcal{B}|-1)-2 e_{G}(\mathcal{B}) \geq 0$ by Lemma 3.4(b), we may use a similar argument to that given in the proof of Claim 5.7 to deduce that there exists $B_{1} \in \mathcal{B}$ with $d_{G}\left(B_{1}\right)=2$. By Claim 5.5, we have

$$
3 \leq\left|B_{1}\right| \leq n-3
$$

Let $p_{1}=u_{1} v_{1}$ and $p_{2}=u_{2} v_{2}$ be the edges in $G$ from $B_{1}$ to $V-B_{1}$, where $u_{1}, u_{2} \in$ $B_{1}$. Let $H_{1}, H_{2}$ be the components of $G-\left\{p_{1}, p_{2}\right\}$ where $V\left(H_{1}\right)=B_{1}$. Let $G_{1}$ be the graph obtained from $H_{1}$ by adding two new vertices $w_{0}, w_{1}$ and edges $p_{3}=$ $u_{1} w_{0}, p_{4}=w_{1} u_{2}, p_{5}=w_{0} w_{1}$. Let $G_{2}$ be the graph obtained from $H_{2}$ by adding a new vertex $w_{2}$ and edges $p_{6}=v_{1} w_{2}, p_{7}=v_{2} w_{2}$, see Fig. 8 .

\section{Claim 5.10}

(a) $G_{1}$ is strong.

(b) $\operatorname{def}(G) \geq \operatorname{def}\left(G_{2}\right)$. 
Proof (a) Suppose that $G_{1}$ is not a brick. Let $\mathcal{B}_{1}$ be the brick partition of $G_{1}$. Then $\left|\mathcal{B}_{1}\right| \geq 2$. Since $G\left[B_{1}\right]$ is strong by Lemma 3.5 and since strong graphs with at least two vertices have minimum degree at least two, we must have $\mathcal{B}_{1}=\left\{B_{1},\left\{w_{0}\right\},\left\{w_{1}\right\}\right\}$. But then $\operatorname{def}\left(G_{1}\right)=\operatorname{def}_{G_{1}}\left(\mathcal{B}_{1}\right)=0$, contradicting the assumption that $G_{1}$ is not a brick.

(b) Consider the brick partition $\mathcal{B}_{2}$ of $G_{2}$. Let $B_{2}$ be the brick of $G_{2}$ which contains $w_{2}$ and $\mathcal{Q}=\mathcal{B}_{2}-\left\{B_{2}\right\} \cup\left\{B_{2} \cup B_{1}\right\}$. Then

$$
\operatorname{def}(G) \geq \operatorname{def}_{G}(\mathcal{Q})=\operatorname{def}_{G_{2}}\left(\mathcal{B}_{2}\right)=\operatorname{def}\left(G_{2}\right) .
$$

We have $\left|V\left(G_{i}\right)\right|<|V(G)|$ for $i \in\{1,2\}$, by (4). Thus, by the minimality of $G$ and Lemma 4.1, there exists a pin-line-generic pin-collinear body-and-pin realization $\left(G_{i}^{*}, q_{i}\right)$ of $G_{i}$ such that $r\left(G_{i}^{*}, q_{i}\right)=2\left(n_{i}+m_{i}\right)-3-\operatorname{def}\left(G_{i}\right)$ for each $i \in\{1,2\}$. Since $r\left(G_{2}^{*}, q_{2}\right)$ is preserved by translations, rotations, and dilations, we may assume that $q_{1}\left(p_{3}\right)=q_{2}\left(p_{6}\right)$ and $q_{1}\left(p_{4}\right)=q_{2}\left(p_{7}\right)$. Define $q: V\left(G^{*}\right) \rightarrow \mathbb{R}^{2}$ by putting $q(x)=q_{1}(x)$ for $x \in V\left(G_{1}^{*}\right)-\left\{w_{0}, w_{1}, p_{3}, p_{4}, p_{5}\right\}, q(x)=q_{2}(x)$ for $x \in V\left(G_{2}^{*}\right)-\left\{w_{2}, p_{6}, p_{7}\right\}, q\left(p_{1}\right)=q_{1}\left(p_{3}\right)=q_{2}\left(p_{6}\right)$, and $q\left(p_{2}\right)=q_{1}\left(p_{4}\right)=q_{2}\left(p_{7}\right)$. Then $\left(G^{*}, q\right)$ is a pin-collinear body-and-pin realization of $G$.

By Claim 5.10(a), $\operatorname{def}\left(G_{1}\right)=0$. Thus $r\left(G_{1}^{*}, q_{1}\right)=2\left(n_{1}+m_{1}\right)-3$, and $\left(G_{1}^{*}, q_{1}\right)$ is infinitesimally rigid. Hence $r\left(G_{1}^{*}+p_{3} p_{4}, q_{1}\right)=r\left(G_{1}^{*}, q_{1}\right)$. Let $F_{1}=G_{1}^{*}-$ $\left\{w_{0}, w_{1}, p_{4}\right\}$ and $F_{2}=G_{2}^{*}-\left\{w_{2}\right\}$. For $i=1,2$, let $t_{i}$ be the restriction of $q_{i}$ to $V\left(F_{i}\right)$. Using Lemma 2.3, we may deduce that $r\left(F_{1}, t_{1}\right)=2\left(n_{1}+m_{1}\right)-3-6=$ $r\left(F_{1}+p_{3} p_{4}, t_{1}\right)$ and $r\left(F_{2}, t_{2}\right)=2\left(n_{2}+m_{2}\right)-3-\operatorname{def}\left(G_{2}\right)-2$. Now Lemma 2.6 implies that

$$
\begin{aligned}
r\left(G^{*}+p_{1} p_{2}, q\right) & =r\left(F_{1}+p_{3} p_{4}, t_{1}\right)+r\left(F_{2}, t_{2}\right)-1 \\
& =2\left(n_{1}+m_{1}\right)+2\left(n_{2}+m_{2}\right)-\operatorname{def}\left(G_{2}\right)-15 .
\end{aligned}
$$

Since $r\left(F_{1}, t_{1}\right)=r\left(F_{1}+p_{3} p_{5}, t_{1}\right)$, we have $r\left(G^{*}, q\right)=r\left(G^{*}+p_{1} p_{2}, q\right)$. Since $n=$ $n_{1}+n_{2}-3$ and $m=m_{1}+m_{2}-3$, this gives

$$
r\left(G^{*}, q\right)=2(n+m)-3-\operatorname{def}\left(G_{2}\right) \geq 2(n+m)-3-\operatorname{def}(G),
$$

by Claim 5.10(b). This contradicts the choice of $G$ and completes the proof of the theorem.

We next extend Theorem 5.1 to multigraphs. A multigraph $H$ is reducible if it can be reduced to a single vertex by repeatedly contracting parallel edges, i.e. pairs of vertices $u, v$ with $d(u, v) \geq 2$. Since these pairs induce strong subgraphs, it follows from Lemma 3.6 that reducible graphs are strong. The next lemma is not difficult to verify by induction on the number of contractions.

Lemma 5.11 Let $G$ be a reducible multigraph. Then $G$ has an infinitesimally rigid body-and-pin realization $\left(G^{*}, q\right)$ in which all pins are collinear.

Theorem 5.12 Let $G=(V, P)$ be a connected multigraph on at least two vertices. Then there exists a pin-collinear body-and-pin realization $\left(G^{*}, q\right)$ of $G$ as a bar-andjoint framework such that $r\left(G^{*}, q\right)=2(|V|+|P|)-3-\operatorname{def}(G)$. 
Proof By Lemma 5.11 we may assume that $G$ is not reducible. Observe that the maximal reducible subgraphs $G_{1}, G_{2}, \ldots, G_{z}$ of $G$ are pairwise vertex-disjoint induced subgraphs. Let $H$ be the multigraph obtained from $G$ by contracting each subgraph $G_{i}$ into a vertex $g_{i}, 1 \leq i \leq z$. Then $H$ is a graph, without multiple edges and isolated vertices, which satisfies $\operatorname{def}(H)=\operatorname{def}(G)$ by Lemma 3.6. Theorem 5.1 implies that there is a pin-collinear body-and-pin realization $\left(H^{*}, q^{\prime}\right)$ of $H$ as a bar-and-joint framework with $r\left(H^{*}, q^{\prime}\right)=2\left|V\left(H^{*}\right)\right|-3-\operatorname{def}(G)$. Let $L_{i}^{\prime}$ denote the pin-line of $g_{i}$ in $\left(H^{*}, q^{\prime}\right), 1 \leq i \leq z$.

It follows from Lemma 5.11 that each subgraph $G_{i}$ has an infinitesimally rigid body-and-pin realization $\left(G_{i}^{*}, q_{i}\right)$ in which all pins are on the same line $L_{i}, 1 \leq i \leq z$. Note that no vertex $v \in V\left(G_{i}\right)$ can have $q_{i}(v)$ on $L_{i}$. By translating and rotating the frameworks $\left(G_{i}^{*}, q_{i}\right)$, if necessary, we may suppose that $L_{i}^{\prime}=L_{i}$ for $1 \leq i \leq z$. Let $p \in E_{H}\left(g_{i}\right)$ for some $1 \leq i \leq z$. Then $p$ corresponds to an edge joining some vertex $u \in V\left(G_{i}\right)$ to a vertex $v \in V\left(G_{j}\right)$ for some $j \neq i$. We may add the pin-vertex $p$ to $\left(G_{i}^{*}, q_{i}\right)$ by performing a 0 -extension which places $p$ at the point $q^{\prime}(p)$ on the line $L_{i}$ and adds edges $u p$ and $p_{u} p$ for some $p_{u} \in N_{G_{i}^{*}}(u)$. We repeat this operation for all $p \in E_{H}\left(g_{i}\right)$ and all $1 \leq i \leq z$ and denote the resulting extended frameworks by $\left(H_{i}, q_{i}^{\prime}\right)$. Note that each of the extended frameworks is infinitesimally rigid by Lemma 2.3.

We can now obtain the desired realization $\left(G^{*}, q\right)$ of $G$ from $\left(H^{*}, q^{\prime}\right)$ by glueing each of the frameworks $\left(H_{i}, q_{i}^{\prime}\right)$ to $\left(H^{*}, q^{\prime}\right)$ along their common pins, then deleting the vertices $g_{i}, 1 \leq i \leq z$, and then adding some extra edges. Since the first two operations replace the infinitesimally rigid subframework induced by $g_{i}$ and its neighbours in $H^{*}$ by the infinitesimally rigid subframework $\left(H_{i}, q_{i}^{\prime}\right)$, it is not difficult to see that the degree of freedom of the resulting framework $\left(G^{*}, q\right)$ is not bigger than that of $\left(H^{*}, q^{\prime}\right)$. Thus we have $r\left(G^{*}, q\right) \geq 2\left|V\left(G^{*}\right)\right|-3-\operatorname{def}(G)=$ $2(|V|+|P|)-3-\operatorname{def}(G)$. The theorem now follows by applying Lemma 3.2.

Theorem 1.4 follows from Theorem 5.12 by putting $\operatorname{def}(G)=0$. Note that Theorem 5.12 also implies that equality can be attained in Lemma 3.2. Thus the "generic rank" of the body-pin graph $G^{*}$ of $G=(V, P)$ is equal to $2(|V|+|P|)-3-\operatorname{def}(G)$.

\section{Maximum Rank Body-and-Pin Frameworks}

A 2-dimensional body-and-pin framework is a pair $(G, q)$ where $G=(V, P)$ is a multigraph and $q$ is a map which associates a point in $\mathbb{R}^{2}$ with each edge $p \in P$. Formal definitions for a $2|P| \times 3|V|$ rigidity matrix $R_{B P}(G, q)$ and for the infinitesimal rigidity of the body-and-pin framework $(G, q)$ are given in $[2,5]$. The rank of $(G, q)$, $r_{B P}(G, q)$, is defined to be the rank of its rigidity matrix $R_{B P}(G, q)$. We show in [2] that if $\left(G^{*}, q\right)$ is a body-and-pin realization of $G$ as a bar-and-joint framework and if $\left.q\right|_{P}$ is the restriction of $q$ to $P$, then the null space of $R\left(G^{*}, q\right)$ is isomorphic to the null space of $R_{B P}\left(G,\left.q\right|_{P}\right)$. This allows us to use Theorem 1.4 to deduce Theorem 1.3. We may also use Theorem 5.12 to deduce:

Theorem 5.13 Let $G=(V, P)$ be a multigraph with no isolated vertices. Then there exists a body-and-pin framework $(G, q)$ such that $r_{B P}(G, q)=3|V|-3-\operatorname{def}(G)$ and, for each $v \in V$, the points $q(p), p \in E_{G}(v)$, are collinear. 
A body-and-pin framework $(G, q)$ is independent if the rows of $R_{B P}(G, q)$ are linearly independent, i.e. $r_{B P}(G, q)=2|P|$. By Theorem 3.1(a), $2|P|=3|V|-3-$ $\operatorname{def}(G)$ if and only if $2 G$ can be covered by three forests. Thus Theorem 5.13 implies the result of Whiteley [15, Theorem 5.4] that, if $2 G$ can be covered by three forests, then $G$ has a realization as an independent body-and-pin framework $(G, q)$ in $\mathbb{R}^{2}$ with the points $q(p), p \in E_{G}(v)$, collinear for each $v \in V$.

\section{Concluding Remarks}

In the body-and-hinge frameworks investigated so far in this paper, each hinge is shared by exactly two bodies. We can obtain more general structures by relaxing this condition.

An identified body-and-hinge framework in $\mathbb{R}^{d}$ is an ordered pair $(H, q)$ where $H=(V \cup P, I)$ is a bipartite graph and $q$ is a map which associates a $(d-2)$ dimensional affine subspace $q(p)$ with each $p \in P$. Infinitesimal motions and infinitesimal rigidity of $(H, q)$ are defined in an analogous way as for body-and-hinge frameworks. Tay and Whiteley [12, Conjecture 2, Theorem 3] give a conjectured characterization of when a bipartite graph has an infinitesimally rigid realization as a $d$-dimensional identified body-and-hinge framework and point out that their conjecture holds when $d=2$. Indeed we may use the rank formula for the 2-dimensional generic (bar-and-joint) rigidity matroid given by Lovász and Yemini in [7] to determine the maximum rank of a realization of a bipartite graph as a 2-dimensional identified body-and-hinge framework. To see this we extend the definition of a bodyand-pin graph.

Let $H=(V \cup P, I)$ be a bipartite graph without isolated vertices. The identified body-and-pin graph of $H$ is the graph $H^{B P}$ with $V\left(H^{B P}\right)=V \cup P$ and

$$
E\left(H^{B P}\right)=\{v p: v \in V, p \in P, v p \in I\} \cup\left\{p_{1} p_{2}: v \in V \text { and } p_{1}, p_{2} \in E_{H}(v)\right\} .
$$

(This definition extends the earlier definition for a graph $G$ by taking $H$ to be the bipartite graph obtained by subdividing each edge of $G$. We then have $G^{*}=H^{B P}$.) Let $\mathcal{F}$ be a partition of $V$. For each $p \in P$, let $w_{\mathcal{F}}(p)$ be the number of sets $F \in \mathcal{F}$ for which $N_{H}(p) \cap F \neq \emptyset$. Put $\operatorname{def}_{H}(\mathcal{F})=3(|\mathcal{F}|-1)-2\left(\sum_{p \in P}\left(w_{\mathcal{F}}(p)-1\right)\right)$ and let $\operatorname{def}(H)=\max \mathcal{F}\left\{\operatorname{def}_{H}(\mathcal{F})\right\}$. By using the above mentioned rank formula for the 2dimensional generic rigidity matroid it is not difficult to show that $r\left(H^{B P}\right)=2(|V|+$ $|P|)-3-\operatorname{def}(H)$.

We believe that Theorem 5.12 can be extended to identified body-and-pin graphs.

Conjecture 6.1 Let $H$ be a bipartite graph. Then there exists a pin-collinear realization $\left(H^{B P}, q\right)$ of $H^{B P}$ such that $r\left(H^{B P}, q\right)=r\left(H^{B P}\right)$.

Note that every identified body-and-pin graph has a pin-collinear realization, which can be obtained for example by putting all pins on the same pin-line.

An affirmative answer to Conjecture 6.1 for the special case where $H$ has a realization as an independent body-and-pin framework follows from the above mentioned result of Whiteley [15, Theorem 5.4]. Whiteley also formulated a similar conjecture to Conjecture 6.1 for 3-dimensional frameworks in [15, p. 93]. 
Acknowledgements We would like to thank Walter Whiteley and Zoltán Szabadka for helpful conversations regarding the relationship between body-and-hinge and bar-and-joint frameworks and the referees for their helpful comments.

\section{References}

1. Crapo, H., Whiteley, W.: Statics of frameworks and motions of panel structures, a projective geometric introduction. Topol. Struct. 6, 43-82 (1982)

2. Jackson, B., Jordán, T.: Pin-collinear body-and-pin frameworks and the molecular conjecture. EGRES TR-2006-06. www.cs.elte.hu/egres/

3. Jackson, B., Jordán, T.: Rigid components in molecular graphs. Algorithmica 48(4), 399-412 (2007)

4. Jackson, B., Jordán, T.: On the rigidity of molecular graphs. Combinatorica (to appear). See also: EGRES TR-2006-02. www.cs.elte.hu/egres/

5. Jackson, B., Jordán, T.: The generic rank of body-bar-and-hinge frameworks. Eur. J. Comb. (to appear). See also: EGRES TR-2007-06. www.cs.elte.hu/egres/

6. Jackson, B., Jordán, T.: Brick partitions of graphs. Discrete Math. (to appear). See also: EGRES TR2007-05. www.cs.elte.hu/egres/

7. Lovász, L., Yemini, Y.: On generic rigidity in the plane. SIAM J. Algebr. Discrete Methods 3, 91-98 (1982)

8. Nash-Williams, C.St.J.A.: Edge-disjoint spanning trees of finite graphs. J. Lond. Math. Soc. 36, 445450 (1961)

9. Nash-Williams, C.St.J.A.: Decomposition of finite graphs into forests. J. Lond. Math. Soc. 39, 12 (1964)

10. Schrijver, A.: Combinatorial Optimization. Springer, Berlin (2003)

11. Tay, T.S.: Rigidity of multi-graphs I Linking Bodies in $n$-space. J. Comb. Theory B 26, 95-112 (1984)

12. Tay, T.S., Whiteley, W.: Recent advances in the generic rigidity of structures. Topol. Struct. 9, 31-38 (1984)

13. Tutte, W.T.: On the problem of decomposing a graph into $n$ connected factors. J. Lond. Math. Soc. 36, 221-230 (1961)

14. Whiteley, W.: The union of matroids and the rigidity of frameworks. SIAM J. Discrete Math. 1(2), 237-255 (1988)

15. Whiteley, W.: A matroid on hypergraphs, with applications in scene analysis and geometry. Discrete Comput. Geom. 4, 75-95 (1989)

16. Whiteley, W.: Some matroids from discrete applied geometry. In: Bonin, J.E., Oxley, J.G., Servatius, B. (eds.) Matroid Theory, Seattle, WA, 1995. Contemp. Math., vol. 197, pp. 171-311. Am. Math. Soc., Providence (1996)

17. Whiteley, W.: Rigidity of molecular structures: geometric and generic analysis. In: Thorpe, M.F., Duxbury, P.M. (eds.) Rigidity Theory and Applications, pp. 21-46. Kluwer Academic, Dordrecht (1999)

18. Whiteley, W.: Counting out to the flexibility of molecules. Physical Biology 2, S116-S126 (2005) 